\title{
Isolation and Gene Expression Analysis of Flowering-related Genes in Lettuce
}

\section{(Lactuca sativa L.)}

\author{
Machiko Fukuda*, Yosuke Yanai, Yuka Nakano, Hidekazu Sasaki, Atsuko Uragami \\ and Kunihiko Okada
}

NARO Institute of Vegetable and Floriculture Science, Tsukuba 305-8666, Japan

Lettuce (Lactuca sativa L.) bolting is often limiting in agricultural production as stem elongation followed by flower bud differentiation results in unmarketable plants. Thus, to solve this problem, it is important to elucidate the molecular mechanisms controlling flowering. To investigate the relationship between gene expression and flowering in lettuce, homologs of flowering-related genes were isolated and their expression over time was analyzed in three cultivars and in one breeding line by using quantitative real-time PCR. In plants grown in the field, the expression of the lettuce homologues APETALA 1 (LsAP1L) and LEAFY (LSLFYL) in the shoot apex correlated with flower bud formation, and FLOWERING LOCUS T (LSFT) expression increased during the transition from vegetative to reproductive growth. The transcription of FLOWERING LOCUS D (LSFLDL), FVE (LSFVEL), and LUMINIDEPENDENS (LSLDL) also increased with flowering. Our previous study showed that $L s F T$ was upregulated during lettuce flowering induced under controlled high temperature conditions. Results from both studies suggest that $L s F T$ is involved in lettuce flowering, both in natural and controlled conditions. This is also the first report on the expression of other flowering-related genes along with flowering of lettuce grown in the field.

Key Words: $L s F L D L, L s F T, L s F V E L, L s L D L$.

\section{Introduction}

Lettuce (Lactuca sativa L.) is one of the most popular leafy vegetables, and it is harvested and consumed during its vegetative growth. High temperature induces the transition from vegetative to reproductive growth in lettuce (Thompson and Knott, 1933), followed by rapid stem elongation and flowering, which is commonly known as bolting. Preventing stalk elongation in lettuce cultivation is essential because the resulting plants are not suitable for commerce. The development of a cultivation technique that adjusts to hot conditions or the breeding of bolting-resistant cultivars would be useful in stable lettuce production and may be achievable once the mechanism determining bolting is understood. In addition to higher temperature, gibberellin (GA), a plant hormone, is involved in lettuce flowering

Received; July 13, 2016. Accepted; September 26, 2016. First Published Online in J-STAGE on October 29, 2016.

Parts of this paper were presented at the 2014 spring meeting of the Japanese Society for Horticultural Science.

* Corresponding author (E-mail: fukudama@affrc.go.jp).
(Wittwer and Bukovac, 1957). We reported that bioactive $\mathrm{GA}_{1}$ increased with the upregulation of L. sativa GA 3-oxidase gene, LsGA3oxl, in lettuce stems elongating at high temperature (Fukuda et al., 2009). Although there are some options to control LsGA3oxl expression or LsGA3ox1 activity to manage lettuce bolting, neither the gene nor the enzyme is fully inhibited because GA also plays important roles in several other developmental events (Yamaguchi, 2008). Moreover, the disruption of $L s G A 3 o x 1$ by Tnt1, a retrotransposon from tobacco, led to a dwarf phenotype, but plants eventually flowered (Mazier et al., 2007) suggesting that different pathway(s) may be acting to induce lettuce flowering. Therefore, further essential factors related to flowering must be considered to achieve fundamental bolting control.

As mentioned above, temperature is the most important environmental signal for inducing flowering in lettuce. Still, lettuce plants are able to flower at relatively low temperatures, although the time taken to do so is longer than at high temperatures (Hiraoka, 1967a, b). We have been investigating the molecular mechanism of lettuce flowering, especially focusing on the 
pathways related to temperature and endogenous developmental stages. Although information on lettuce flowering is quite limited, important progress has been made in understanding the molecular mechanisms that control flowering in Arabidopsis spp., as well as in other plants (e.g., Amasino, 2010; Glover, 2014). In Arabidopsis spp., the initiation of flowering is mainly controlled through the following pathways: photoperiod, vernalization, GA, autonomous regulation, and age (Srikanth and Schmid, 2011). Multiple floral inductive cues are integrated into a set of flowering time integrator genes, including the SUPPRESSOR OF OVEREXPRESSION OF CONSTANS 1 (Lee et al., 2000; Samach et al., 2000) and the FLOWERING LOCUS T (FT) (Kardailsky et al., 1999; Kobayashi et al., 1999). These integrator genes act upstream of the genes involved in floral morphogenesis, such as APETALA1 (AP1) (Mandel et al., 1992) and $L E A F Y(L F Y)$ (Weigel et al., 1992). Among the pathways related to temperature and endogenous growth, the autonomous pathway genes promote flowering by suppressing FLOWERING LOCUS C (Koornneef et al., 1991), which acts as the master regulator of the vernalization pathway (Michaels and Amasino, 1999). Among the autonomous pathway components, FLOWERING LOCUS D (FLD) and FVE are similar to the components of the mammalian histone deacetylase corepressor complexes 1 and 2, which are involved in the repression of gene expression via deacetylation of histone residues (Ausín et al., 2004; He et al., 2003). LUMINIDEPENDENS (LD) encodes a homeodomain protein (Lee et al., 1994).

Downstream of flowering pathways, there are also important floral integrators. Among them, FT is generally expressed in leaf phloem (Takada and Goto, 2003). Grafting experiments demonstrated that floral stimuli travel long distances (Notaguchi et al., 2008), and that $F T$ is a main trigger of floral initiation. Reports on FT genes isolated from several plants have shown that they are involved in flowering (Hayama et al., 2007; Lifschitz et al., 2006; Oda et al., 2012; Vergara et al., 2016; Xia et al., 2012). In a previous study, we isolated and characterized the L. sativa FT homolog ( $L s F T$ ) (Fukuda et al., 2011). As LsFT overexpression induced early flowering in transgenic $A$. thaliana, $L S F T$ seems to have a similar function to $F T$ in the floral induction pathway. The expression of $L s F T$ was abundant in the largest lettuce leaves, and upregulated during stem rapid elongation and flower bud differentiation in controlled high temperature conditions (Fukuda et al., 2011). These results revealed that $L s F T$ expression could be related to floral induction in lettuce plants grown in experimental conditions. However, the lettuce cultivar used in our previous study was a leafy type, which has been used in physiological studies (Hsiao and Vidaver, 1971; Toyomasu et al., 1998) and it is also important to consider different varieties, including the major type used in lettuce production, i.e., heading lettuce (Ryder, 1999). The present study aimed to evaluate the expression of flowering-related genes during the lettuce flowering stage in natural conditions. Hence, the relationship between flowering time and $L s F T$ gene expression was evaluated, along with the expression patterns of homologs of other putative flowering-related genes, by using several lettuce cultivars and breeding lines with different flowering traits.

\section{Materials and Methods}

\section{Plant materials}

Experiments were conducted in the field at the NARO Institute of Vegetable and Floriculture Science (NIVFS) in Tsukuba, Japan (36 $\left.6^{\prime} 45^{\prime \prime} \mathrm{N}, 140^{\circ} 6^{\prime} 14^{\prime \prime} \mathrm{E}\right)$. The L. sativa 'Leaf Lettuce Green' ('LLG') (Sakata Seed Corporation, Yokohama, Japan), 'Texas Green' ('TG'), and 'Patriot' ('Pat') (Nitto Nosan, Yokohama, Japan), and a lettuce breeding line (BL) were used in this study. The cultivar 'LLG' is a leafy, early bolting, non-heading lettuce whereas the other cultivars and breeding line are heading lettuces; among the heading lettuces, 'TG' bolts earlier than 'Pat', which is one of the latest bolting cultivars. BL was developed by crossing L. sativa with its wild relative Lactuca virosa L., followed by selfing (Noguchi, unpublished), and it bolts later than 'Pat'. Seeds from cultivars and the breeding line were sown on April 1, 2013 in cell trays filled with soil, and kept inside a glasshouse where temperature and photoperiod were not controlled. Meanwhile, the field was fertilized with chemical NPK fertilizer (converted to $18 \mathrm{~kg}$ nitrogen, $18 \mathrm{~kg}$ phosphorus, and $18 \mathrm{~kg}$ potassium/10 a) and covered with white agricultural mulch. Sixty-four seedlings were planted in two rows, separated by $25 \mathrm{~cm}$, in an open field on May 2, 2013 and grown until July 17, 2013. Air temperature and daily precipitation data acquired from the Weather Data Acquisition System, Institute for Agro-Environmental Science (http://www.niaes.affrc.go.jp/niaesaws/) (at $36^{\circ} 12^{\prime \prime} \mathrm{N}, 140^{\circ} 6^{\prime} 41^{\prime \prime}$ E) were used. Weather conditions during the cultivation period are shown in Figure 1. After harvesting, the shoot apex of each plant was stained with methyl blue and observed under a stereomicroscope (Leica Microsystems, Wetzlar, Germany) using a magnification ranging from 6.3 to $25 \mathrm{x}(10 \mathrm{x}$ eyepieces and $0.63-2.5 \mathrm{x}$ objectives). Flowering scores were defined as 0 (vegetative), 1 (apical dome enlarging), 2 (first inflorescence forming), or 3 (lateral inflorescence forming) (Fig. 2).

\section{Isolation of flowering-related genes from lettuce}

Total RNA was isolated from lettuce 'LLG' leaves using an RNA Plant Mini Kit and treated with RNasefree DNase (both from Qiagen, Hilden, Germany). First-strand cDNA was prepared using a PrimeScript RT reagent Kit (TAKARA Bio Inc., Kusatsu, Japan) and $0.5 \mu \mathrm{g}$ DNase-treated RNA. PCR was performed for each gene with degenerate and nested primers that 


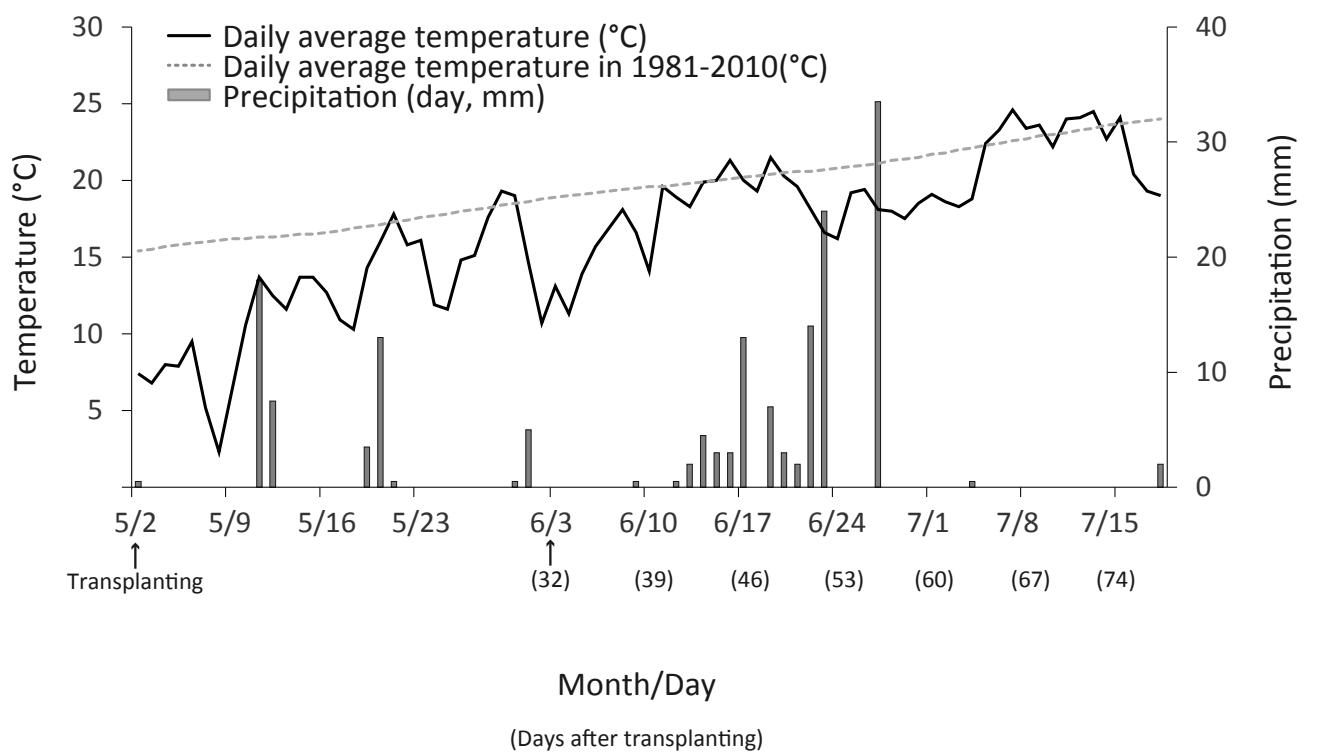

Fig. 1. Average air temperature and precipitation in the field at the NARO Institute of Vegetable and Floriculture Science, Tsukuba, Japan. Seeds were sown on April 1, 2013 and the seedlings were transplanted on May 2, 2013.

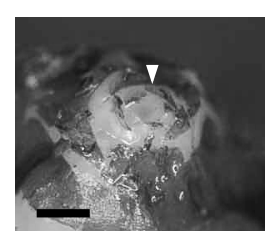

0 , Vegetative

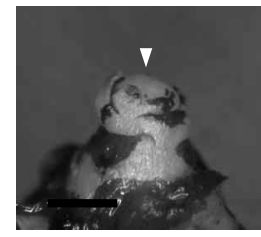

1, Apical dome enlarging

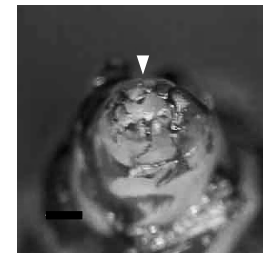

2, First inflorescence forming

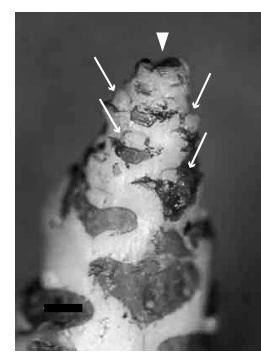

3, Lateral inflorescences forming

Fig. 2. Lettuce shoot apex morphology used for defining flowering scores. Lettuce ('LLG') shoot apexes were stained with methyl blue and observed under the stereomicroscope (magnification $=6.3-25 \mathrm{x}$ ). Flowering scores were defined as: 0 , vegetative; 1 , apical dome enlarging; 2, first inflorescence forming; and 3, lateral inflorescences forming. Arrowheads indicate the apical meristem. Arrows indicate the lateral meristem. Scale bar: $0.5 \mathrm{~mm}$.

were specifically designed based on known mRNA sequences for each gene from several plant species (Table 1). PCR fragments were cloned into a pGEM-T easy vector (Promega, Madison, Wisconsin, USA) or into a pCR-Blunt II-TOPO vector (Thermo Fisher Scientific Inc., Waltham, Massachusetts, USA). Eight clones were sequenced using a BigDye terminator cycle sequencing kit in a 3130 DNA Analyzer (both from Thermo Fisher Scientific Inc.).

Rapid amplifications of cDNA ends (RACE) reactions were performed using a Marathon cDNA amplification kit and an Advantage cDNA PCR kit (both from TAKARA Bio Inc.), following the manufacturer's instructions and using gene-specific sense and antisense primers (Table 1). Amplification products were cloned into pGEM-T easy vectors and sequenced. End-to-end PCR was carried out to obtain the full-length lettuce cDNA, and amplified PCR fragments were cloned into a PCR-Blunt-TOPO vector (Thermo Fisher Scientific Inc.). Eight clones were sequenced.

\section{Sequence analysis}

Sequences of flowering-related genes were identified using the basic local alignment search tool (BLAST) against the National Center for Biotechnology Information (NCBI) database (http://www.ncbi.nlm.nih.gov/ BLAST/), and aligned with homologs sequences using Clustal Omega (http://www.ebi.ac.uk/Tools/msa/clustalo/) and GENETYX (GENETYX CORPORATION, Tokyo, Japan). Sequences have been deposited at the DNA Data Bank of Japan under the accessions LC164344 (LsAP1L), LC164345 (LsLFYL), LC164346 $(L s L D L), \quad \mathrm{LC} 164347 \quad(L S F L D L), \quad$ and $\mathrm{LC} 164348$ (LsFVEL). 
Table 1. Primers used in this study.

\begin{tabular}{|c|c|c|}
\hline Genes & & Sequences \\
\hline \multirow[t]{2}{*}{$L s A P 1 L$ fragment } & Forward & 5'-AGGAGGATAGAGAACAAGATTAACAG-3' \\
\hline & Reverse & 5'-GTGTTCTGCTCYTGTATGGCCTTTCC-3' \\
\hline$L s A P 1 L$ fragment (nested) & Forward & 5'-GATTAACAGRCARGTWACTTTCTCRAAG-3' \\
\hline \multirow{2}{*}{$L s A P 1 L \mathrm{qRT}-\mathrm{PCR}$} & Forward & 5'-CGAAGAGGAGAGGAGGGTTG-3' \\
\hline & Reverse & 5'-AGAGCTTTCCTTTGTTGGAGAAG-3' \\
\hline \multirow[t]{2}{*}{$L s L F Y L$ fragment } & Forward & 5'-ATGTGTGAGAAAATAAAAATGGACCC-3' \\
\hline & Reverse & 5'-AGCACTGCTGCGTTCAGCATGAC-3' \\
\hline$L s L F Y L$ fragment (nested) & Forward & 5'-GAAAATAAAAATGGACCCTGATGCAC-3' \\
\hline \multirow[t]{2}{*}{$L s L F Y L$ qRT-PCR } & Forward & 5'-GCGAAAGAGAGGGTGAAAA-3' \\
\hline & Reverse & 5'-CGAAGCAACCTCATCAAGACA-3' \\
\hline \multirow[t]{2}{*}{$L s F V E L$ fragment } & Forward & 5'-GTBCCCRTTCTSTAHGACTGGC-3' \\
\hline & Reverse & 5'-CCCARATRTTCAARMVACCATC-3' \\
\hline$L s F V E L$ fragment (nested) & Reverse & 5'-TRTTCAARMVACCATCYTCWGC-3' \\
\hline \multirow[t]{2}{*}{ LsFVEL RACE } & Sense & 5'-CGTTTGTTTGATCGTCGAAACCTTAC-3' \\
\hline & Antisense & 5'-ATCAGATCAGGACGCGACTCTGTG-3' \\
\hline \multirow[t]{2}{*}{$L s F V E L$ full-length cDNA } & Forward & 5'-AGAAAAACATAGGGCAGAGAGAGG-3' \\
\hline & Reverse & 5'-TAAATCATAATTGATGTATGATAATGAATC-3' \\
\hline \multirow[t]{2}{*}{$L s F V E L$ qRT-PCR } & Forward & 5'-GATCATATATCAACATTGGTTGG-3' \\
\hline & Reverse & 5'-AGATCCCACGTGGGCCAAG-3' \\
\hline \multirow[t]{2}{*}{$L s L D L$ fragment } & Forward & 5'-AGRGAYTTRWTRAAYCCDAAGGCTG-3' \\
\hline & Reverse & 5'-WAWWRNGCACGCATYTTTGCTTTYTG-3' \\
\hline$L s L D L$ fragment (nested) & Reverse & 5'-CGCATYTTTGCTTTYTGDATATCATC-3' \\
\hline \multirow[t]{2}{*}{$L s L D L \mathrm{qRT}-\mathrm{PCR}$} & Forward & 5'-AGGAGTGGAGTGTGTGTTATGGA-3' \\
\hline & Reverse & 5'-CTTTGGGATTTGCTGGGATTTC-3' \\
\hline \multirow[t]{2}{*}{$L s F L D L$ fragment } & Forward & 5'-AYCARGAYGAYCCRTATGAYATGGG-3' \\
\hline & Reverse & 5'-YADYCTCWTBTCATCRCCHCCTC-3' \\
\hline$L s F L D L$ fragment (nested) & Reverse & 5'-TGHTGRTTRAARTGWGAYTSDAGYTG-3' \\
\hline$L s F L D L$ qRT-PCR & Forward & 5'-AACCTCAAATGCCCGAAGAA-3' \\
\hline \multirow[t]{2}{*}{$L s F T$ qRT-PCR } & Forward & 5'-CGATATACCAGCGACCACGGGAGCA-3' \\
\hline & Reverse & 5'-TGACGCCATCCAGGGGCATACACA-3' \\
\hline \multirow[t]{2}{*}{ Tubulin qRT-PCR } & Forward & 5'-GGCAAAATGAGCACGAAAGAG-3' \\
\hline & Reverse & 5'-GATCCATTCCACAAAGTAAGACGAG-3' \\
\hline
\end{tabular}

For cloning primer design, homolog sequences were used. For AP1: A. thaliana (NM_105581), Brassica rapa L. (XM_009129455), V. vinifera (NM_001281281), Chrysanthemum seticuspe f. boreale (AB679273), and P. trichocarpa (XM_002311317). For LFY: A. thaliana (NM_125579), B. oleracea (Z18362), M. truncatula (XM_003602697), and Solanum lycopersicum L. (AF_197936). For FVE; A. thaliana (AF498102), Dimocarpus longan L. (FJ862913), Glycine max (L) Merr. (NM_001289270), Ipomoea nil L. (DQ250682), and Pisum sativum L. (AY830931). For LD: A. thaliana (NM_001203731), G. max (XM_003553599), M. truncatula (XM_003625840), P. trichocarpa (XM_006386702), and V. vinifera (XM_010653752). For FLD: A. thaliana (AY849997), Brachypodium distachyon (L.) P. Beauv. (XM_014896047), G. max (XM_003520213), Hordeum vulgare L. (AK368737), Oryza sativa L. (AK073751), P. trichocarpa (XM_002311957), V. vinifera (XM_010660054), and Zea may L. (XM_003520213).

Gene expression of flowering-related genes in lettuce

To analyze flowering-related genes, the largest leaves were harvested at each sampling point. Total RNA was isolated from each sample with an RNeasy Plant Mini Kit and treated with RNase-free DNase (both from Qiagen). First-strand cDNA was prepared using a PrimeScript RT reagent Kit (TAKARA Bio Inc.) with $0.5 \mu \mathrm{g}$ of DNase-treated RNA. Quantitative real timePCR (qRT-PCR) was performed in an Mx3000p (Agilent Technologies, Santa Clare, California, USA) using SYBR Premix Ex Taq II (Tli RNaseH Plus) (TAKARA Bio Inc.). Cloned regions of each gene were sequenced in other cultivars and in the breeding line and gene-specific primers were designed based on con- served sequences (Table 1). The absence of unwanted by-products was confirmed by automated melting curve analysis. Transcripts were normalized based on the expression of the tubulin (AB232704) gene.

\section{Results}

Cloning of flowering-related genes from lettuce

A 454-bp fragment of a putative $A P 1$ homolog and a 1218-bp fragment of a putative $L F Y$ homolog were isolated and named $L S A P 1 L$ and $L S L F Y L$, respectively. LSAPIL showed $77.3 \%$ homology with Vitis vinifera L. AP1, 77.3\% with Populus trichocarpa Torr. \& A. Gray ex. Hook. $A P 1$, and $77.2 \%$ with A.thaliana $A P 1$. LSLFYL shared $71.9 \%$ identity with Medicago 
A

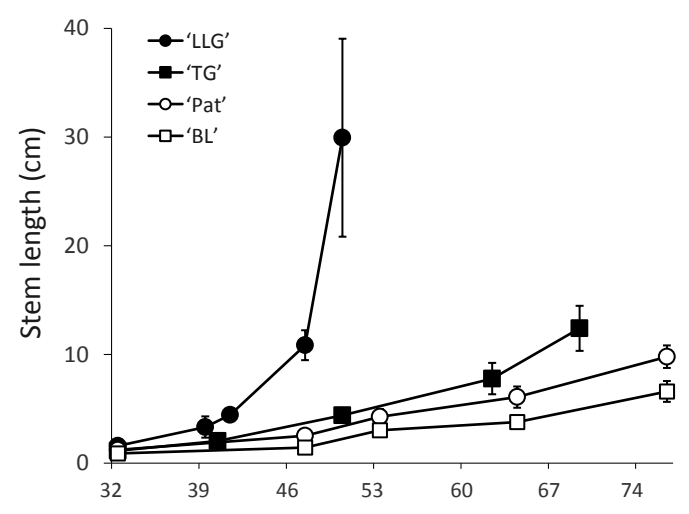

B

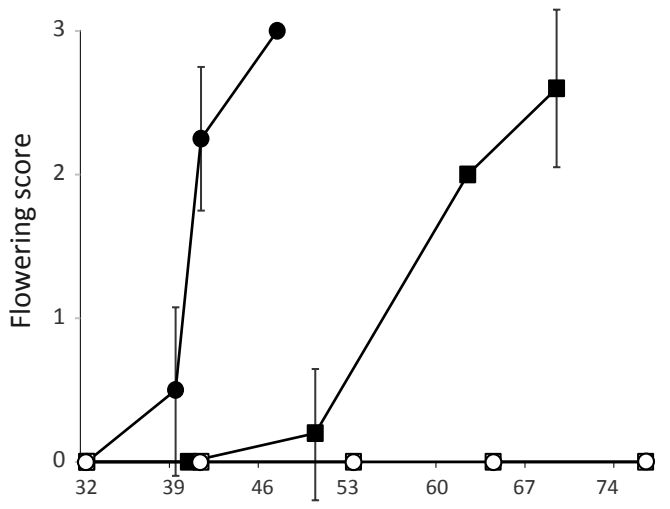

Days after transplanting

Fig. 3. Changes in stem length (A) and flowering scores (B) of lettuce plants grown in the field. Lettuce cultivars: 'LLG' (solid circle), 'TG' (solid square), 'Pat' (open circle), and BL (open square). Values are the means of five plants ( \pm SD). Flowering scores are: 0 , vegetative; 1 , apical dome enlarging; 2, first inflorescence forming; and 3, lateral inflorescences forming.

truncatula Gaertn. $L F Y, 67.3 \%$ with A. thaliana $L F Y$, and $67.3 \%$ with Brassica oleracea L. LFY.

The open reading frame (ORF) of the putative fulllength FVE homolog isolated from L. sativa (LSFVEL) encoded 486 amino acids, and was very similar to that of A. thaliana (74.3\%), Ipomoea nil (L.) Roth (73.8\%), and Glycine $\max (\mathrm{L}$.$) Merr. (73.4\%). The 421$ amino acid sequence of the putative protein encoded by $L S L D L \quad$ cDNA showed 51\% homology with P. trichocarpa $L D, 50 \%$ with $G$. max $L D$, and $44 \%$ with A. thaliana $L D$. The putative FLD homolog ( $L S F L D L)$ had 635-bp and its ORF had 58\% homology with $V$ vinifera FLD, 58\% with P. trichocarpa FLD, and $55 \%$ with $A$. thaliana FLD. Combining GenBank information and multiple sequence comparisons provided evidence that the putative genes isolated from lettuce were $A P 1, L F Y, F V E, L D$, and $F L D$ homologs.

\section{Phenology of lettuce grown in the field}

Daily air temperatures at Tsukuba increased steadily from May to the end of July (Fig. 1). The average daily temperature during the experiment was $23.2^{\circ} \mathrm{C}$, whereas the average daily temperature during the same period from 1981 to 2010 , was $21.3^{\circ} \mathrm{C}$. 'LLG' stems elongated rapidly in the 41 days following transplantation, and 'TG' stems elongated faster than the stems other heading types, i.e., 'Pat' and $\mathrm{BL}$, which remained under $10 \mathrm{~cm}$ throughout the experiment (Fig. 3A). Flower bud differentiation was only observed in 'LLG' and 'TG' (Fig. 3B) and 'LLG' presented flowering scores over 1 earlier than ' $\mathrm{TG}$ ' (41 and 62 days after transplanting, respectively). The stem elongation observed in 'LLG' and ' $\mathrm{TG}$ ' during the experiment is shown in Figure 4A and $\mathrm{B}$. At the end of the field experiment, i.e., 76 days after transplanting, 'LLG' flowered (Fig. 4C), whereas the flower stalk of 'TG' was still elongating (Fig. 4D).

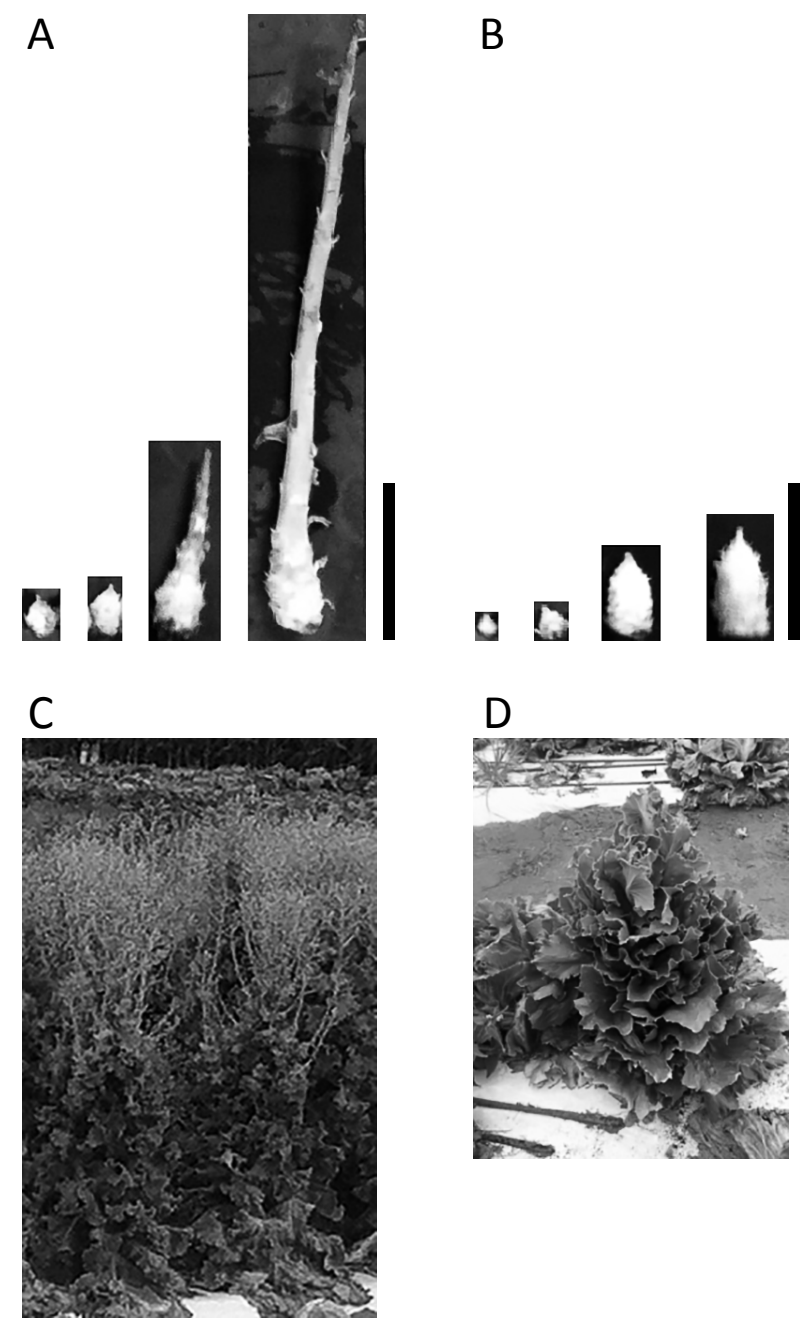

Fig. 4. Flowering phenotypes of 'LLG' and 'TG'. Stem elongation observed in 'LLG' (A) and 'TG' (B) during the experiment. Seventy-six days after transplanting (at the end of the experiment), 'LLG' flowered (C) whereas the flower stalk of 'TG' was still elongating (D). Scale bar in A and B: $10 \mathrm{~cm}$. 
Expression analysis of flowering-related genes in lettuce grown in the field

To investigate changes in gene expression during the transition from vegetative to reproductive growth, $L S A P I L$ and $L S L F Y L$ expressions were measured in the shoot tips. As shown in Figure 5, a drastic upregulation of $L S A P I L$ and $L S L F Y L$ was observed in 'LLG' at day 47 after transplanting and in 'TG' at day 69 after transplanting. The expression patterns of these genes corresponded to flowering scores $>2$ and showed the progress of flower bud formation in 'LLG' and ' $T G$ ' in terms of the expression level of the genes identified in flower buds.

In our previous study, we found that lettuce FT homolog (LsFT) transcripts were most abundant in the largest leaves. Thus, we sequentially harvested the largest lettuce leaves and evaluated the level of $L S F T$ transcripts within them. Substantial increases were noticed in 'LLG' 41 days after transplanting and in ' $T G$ ' 62 days after transplanting, whereas 'Pat' and BL showed only a slight increase in $L s F T$ transcript levels (Fig. 6).

The gene expression of flowering-related genes in lettuce, determined by qRT-PCR, allowed classifying

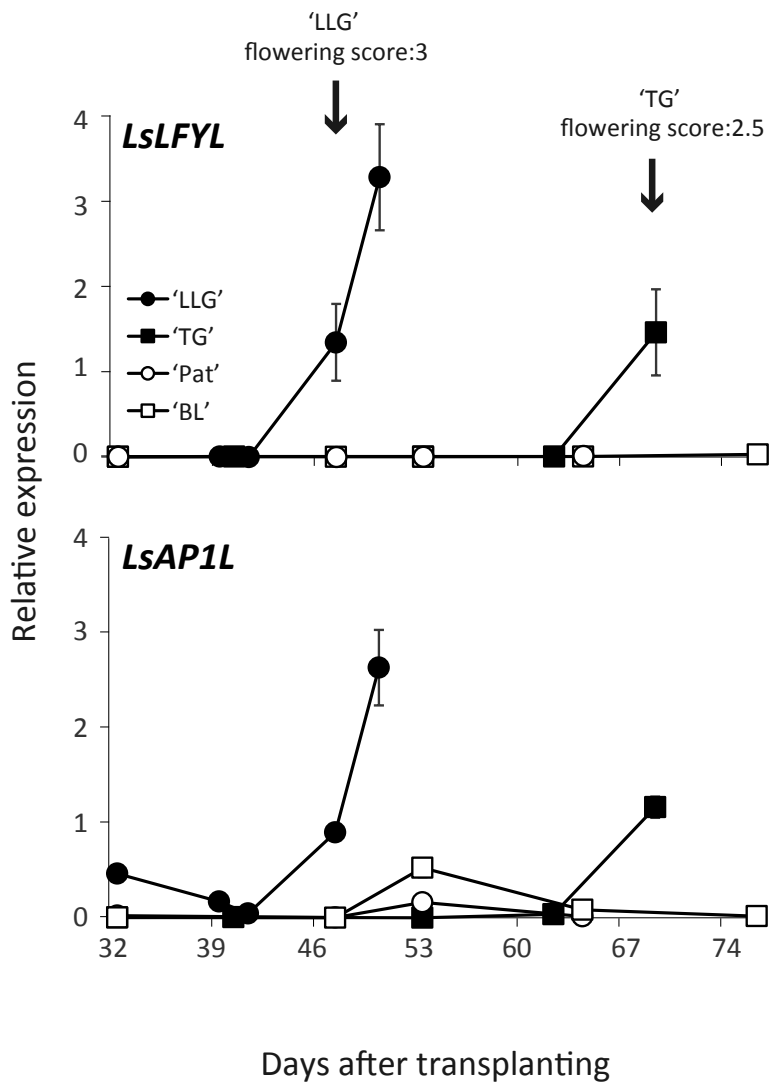

Fig. 5. Expression patterns of $L S L F Y L$ and $L S A P I L$. The number of days needed to detect $L S L F Y L$ and $L s A P I L$ expression in the shoot apex were determined by quantitative real time-PCR. Lettuce cultivars and the breeding line were grown in the field and samples were collected sequentially from June 3 to July 17, 2013. Values shown are the means \pm SD of three biological replicates.
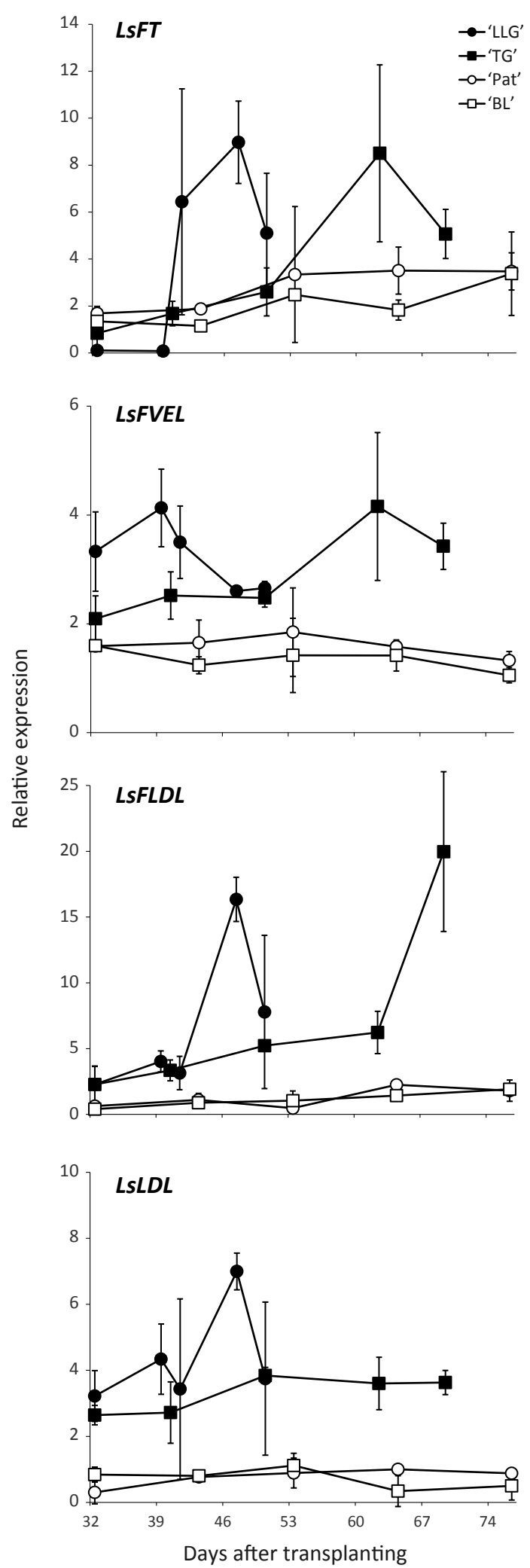

Fig. 6. Expression patterns of $L s F T, L s F V E L, L s F L D L$, and $L s L D L$ in field-grown lettuce plants. The number of days needed to detect $L S F T, L S F V E L, L S F L D L$, and $L S L D L$ expression in the largest lettuce leaves was determined by quantitative real timePCR. Lettuce cultivars and the breeding line were grown in the field, and samples were collected sequentially from June 3 to July 17, 2013. Values shown are the means \pm SD of three biological replicates. 
gene expression patterns into three groups: 1) high gene expression in early flowering cultivars and low gene expression in late flowering cultivars; 2) gene expression increased as flowering progressed; and 3) gene expression patterns were not associated with flowering. Among them, LsFVEL expression in 'LLG' was high at transplanting, reached a maximum at 39 days after transplanting, and decreased at 47 days after transplanting, when flower buds could be observed without using a microscope. The expression of $L S F V E L$ in 'TG' increased at 62 days after transplanting, when the shoot apex differentiated to form first inflorescences. The peaks of $L S F L D L$ expression in 'LLG' and 'TG' occurred at 47 and 69 days after transplanting, respectively. Although $L s L D L$ transcript levels in 'TG' did not show an obvious increase like that observed in 'LLG', they were both higher than $L s L D L$ levels in 'Pat' and BL (Fig. 6).

\section{Discussion}

The expression patterns of $L F Y$ and $A P 1$ have been studied in A.thaliana, and homologs of these genes have been investigated in several plant species (Benlloch et al., 2007). In A.thaliana, LFY confers flower-meristem identity at an early stage of flower development and has a role in the later activation of floral genes (Parcy et al., 1998). APl expression was detected in floral primordia and subsequent stages in a long-day condition (Wigge et al., 2005), and the activation of $A P 1$ could be mediated through $L F Y$ or $F T$ and $F D$ (Parcy et al., 1998; Ruiz-García et al., 1997). Li et al. (2009) examined the homologs of $L F Y$ and $A P 1$ in Chrysanthemum spp. and showed that the expression of these genes increased under flowering conditions. In our study, the expression patterns of $L S L F Y L$ and LSAPIL in the shoot apex of lettuce grown in the field were correlated with flowering scores (Fig. 5), suggesting that these genes are involved in flower bud formation in lettuce, similar to that reported for several plant species (Berbel et al., 2001; Li et al., 2009; Rottmann et al., 2000; Wada et al., 2002). The activation of FT has been shown to play a central role in the flowering process and is highly conserved in plant species (see references in the Introduction section). Our previous study (Fukuda et al., 2011) demonstrated that LsFT expression could be related to the transition from vegetative to reproductive growth in controlled high temperature conditions which induce lettuce flowering. Experiments in growth cabinets offer certain advantages when applying treatments and replicating experiments under tightly controlled environmental conditions. In contrast, natural environments comprise several factors that are unpredictable and fluctuate. With the growing interest in studying the genetics of adaptation in ecological field environments (Izawa, 2015), several studies have been conducted to demonstrate the advantages of using both natural and con- trolled environments (Champigny et al., 2013; Guevara et al., 2012; Plessis et al., 2015). In the present study, $L s F T$ expression increased in 'LLG' and 'TG' cultivars grown in the field and was correlated with flowering (Figs. 3B and 6). Results indicated that high $L s F T$ expression can be detected during the transition to reproductive growth, even when plants are subject to environmental fluctuations occurring in natural conditions, and that this gene induces flowering.

Although knowledge on the autonomous pathway is still limited, several homologs of the autonomous pathway genes have been mapped in plant genomes (Hecht et al., 2005). Hu et al. (2014) identified the G. max homolog of FLD (GmFLD), and transgenic A. thaliana expressing $G m F L D$ flowered earlier than wild type plants; GmFLD complemented the late flowering phenotype of the fld mutant in A. thaliana. Sun et al. (2012) isolated the FVE homolog gene from Doritaenopsis sp. and showed that its expression levels increased during the transition from vegetative to reproductive growth. The late flowering phenotype associated with $l d$ mutants (Lee et al., 1994; Rédei, 1962) indicated that the $L D$ gene plays an important role in floral induction. However, little is still known, even in A.thaliana. The key function of these autonomous pathway genes in A. thaliana is to promote flowering by repressing the expression of $F L C$, which is epigenetically silenced by vernalization. Although lettuce cultivars do not require vernalization to induce flowering, some Lactuca species have biennial and perennial traits (Lebeda et al., 2006). Our results provided additional information on the existence and function of autonomous pathway genes in the studied lettuce cultivars, which seem to have lost vernalization. The expression patterns of $L s F V E L, L s F L D L$, and $L s L D L$ (Fig. 6) suggested that lettuce homologs of autonomous pathway genes may play important roles in the flowering process, at least under the conditions set in our experiments.

Understanding the genetic basis of gene expression will provide new perspectives on the dynamics of the gene network inducing and responding to flowering. In particular, revealing the genetic factors related to differences in bolting between cultivars would be useful for developing new breeding techniques and new lettuce cultivars. Thus, further studies on flowering-related genes may lead to improvements in cultivars that will perform better in response to environmental changes.

\section{Acknowledgments}

We are grateful to Dr. Yuji Noguchi (NIVFS) for kindly providing the seeds of 'Texas Green ('TG')' and lettuce breeding line (BL) and members of the Tsukuba Technical Support Center (NARO) for preparing the plant material. We also thank Dr. Akio Ohyama (NIVFS) for his advice of this manuscript. 


\section{Literature Cited}

Amasino, R. 2010. Seasonal and developmental timing of flowering. Plant J. 61: 1001-1013.

Ausín, I., C. Alonso-Blanco, J. A. Jarillo, L. Ruiz-García and J. M. Martínez-Zapater. 2004. Regulation of flowering time by FVE, a retinoblastoma-associated protein. Nat. Genet. 36: 162-166.

Benlloch, R., A. Berbel, A. Serrano-Mislata and F. Madueño. 2007. Floral initiation and inflorescence architecture: A comparative view. Ann. Bot. 100: 659-676.

Berbel, A., C. Navarro, C. Ferrandiz, L. A. Canas and F. Madueño. 2001. Analysis of PEAM4, the pea AP1 functional homologue, supports a model for $A P 1$ - like genes controlling both floral meristem and floral organ identity in different plant species. Plant J. 25: 441-451.

Champigny, M. J., W. W. Sung, V. Catana, R. Salwan, P. S. Summers, S. A. Dudley, N. J. Provart, R. K. Cameron, G. B. Golding and E. A. Weretilnyk. 2013. RNA-Seq effectively monitors gene expression in Eutrema salsugineum plants growing in an extreme natural habitat and in controlled growth cabinet conditions. BMC Genomics 14: 578. DOI: 10.1186/1471-2164-14-578. <http://bmcgenomics.biomedcentral.com/articles/10.1186/1471-2164-14-578/>.

Fukuda, M., S. Matsuo, K. Kikuchi, Y. Kawazu, R. Fujiyama and I. Honda. 2011. Isolation and functional characterization of the FLOWERING LOCUS T homolog, the LSFT gene, in lettuce. J. Plant Physiol. 168: 1602-1607.

Fukuda, M., S. Matsuo, K. Kikuchi, W. Mitsuhashi, T. Toyomasu and I. Honda. 2009. The endogenous level of $\mathrm{GA}_{1}$ is upregulated by high temperature during stem elongation in lettuce through LsGA3ox1 expression. J. Plant Physiol. 166: $2077-$ 2084.

Glover, B. 2014. Understanding flowers and flowering second edition. OXFORD UNIVERSITY PRESS, Oxford.

Guevara, D. R., M. J. Champigny, A. Tattersall, J. Dedrick, C. E. Wong, Y. Li, A. Labbe, C.-L. Ping, Y. Wang, P. Nuin, G. B. Golding, B. E. McCarry, P. S. Summers, B. A. Moffatt and E. A. Weretilnyk. 2012. Transcriptomic and metabolomic analysis of Yukon Thellungiella plants grown in cabinets and their natural habitat show phenotypic plasticity. BMC Plant Biol. 12: 175. DOI: 10.1186/1471-2229-12-175. <http:// bmcplantbiol.biomedcentral.com/articles/10.1186/1471-2229$12-175 />$.

Hayama, R., B. Agashe, E. Luley, R. King and G. Coupland. 2007. A circadian rhythm set by dusk determines the expression of FT homologs and the short-day photoperiodic flowering response in Pharbitis. Plant Cell 19: 2988-3000.

He, Y., S. D. Michaels and R. M. Amasino. 2003. Regulation of flowering time by histone acetylation in Arabidopsis. Science 302: 1751-1754.

Hecht, V., F. Foucher, C. Ferrándiz, R. Macknight, C. Navarro, J. Morin, M. E. Vardy, N. Ellis, J. P. Beltrán, C. Rameau and J. L. Weller. 2005. Conservation of Arabidopsis flowering genes in model legumes. Plant Physiol. 137: 1420-1434.

Hiraoka, T. 1967a. Ecological studies on the salad crops. I. Effects of temperature, photoperiod and gibberellin spray on bolting, budding and flowering time of head lettuce (Lactuca sativa L. cultivar. Wayaheead, Edogawa strain). J. Japan. Soc. Hort. Sci. 36: 70-78.

Hiraoka, T. 1967b. Ecological studies on the salad crops. II. Effects of photoperiods on flower bud differentiation, bolting and heading in lettuce, with special reference to the difference of photoperiodic sensibility between varieties on various growing stages. J. Japan. Soc. Hort. Sci. 36: 411420.

Hsiao, A. I. and W. Vidaver. 1971. Water content and phytochrome-induced potential germination responses in lettuce seeds. Plant Physiol. 47: 186-188.

$\mathrm{Hu}$, Q., Y. Jin, H. Shi and W. Yang. 2014. GmFLD, a soybean homolog of the autonomous pathway gene FLOWERING LOCUS D, promotes flowering in Arabidopsis thaliana. BMC Plant Biol. 14: 263. DOI: 10.1186/s12870-014-0263 -x. $<$ http://bmcplantbiol.biomedcentral.com/articles/10.1186/ s12870-014-0263-x/>.

Izawa, T. 2015. Deciphering and prediction of plant dynamics under field conditions. Curr. Opin. Plant Biol. 24: 87-92.

Kardailsky, I., V. K. Shukla, J. H. Ahn, N. Dagenais, S. K. Christensen, J. T. Nguyen, J. Chory, M. J. Harrison and D. Weigel. 1999. Activation tagging of the floral inducer FT. Science 286: 1962-1965.

Kobayashi, Y., H. Kaya, K. Goto, M. Iwabuchi and T. Araki. 1999. A pair of related genes with antagonistic roles in mediating flowering signals. Science 286: 1960-1962.

Koornneef, M., C. J. Hanhart and J. H. van der Veen. 1991. A genetic and physiological analysis of late flowering mutants in Arabidopsis thaliana. Mol. Gen. Genet. 229: 57-66.

Lebeda, A., E. J. Ryder, R. Grube, I. Doležalová and E. Kř́stková. 2006. Lettuce (Asteraceae; Lactuca spp.). p. 377472. In: R. J. Singh (ed.). Genetic resources, chromosome engineering, and crop improvement. CRC Press, Boca Raton, FL.

Lee, I., M. J. Aukerman, S. L. Gore, K. N. Lohman, S. D. Michaels, L. M. Weaver, M. C. John, K. A. Feldmann and R. M. Amasino. 1994. Isolation of LUMINIDEPENDENS: A gene involved in the control of flowering time in Arabidopsis. Plant Cell 6: 75-83.

Lee, H., S. S. Suh, E. Park, E. Cho, J. H. Ahn, S. G. Kim, J. S. Lee, Y. M. Kwon and I. Lee. 2000. The AGAMOUS-IIKE 20 MADS domain protein integrates floral inductive pathways in Arabidopsis. Genes Dev. 14: 2366-2376.

Li, L., T. Niki, T. Nishijima, M. Douzono, M. Koshioka and T. Hisamatsu. 2009. Roles of $\mathrm{CmFL}, \mathrm{CmAFLl}$, and $\mathrm{CmSOCl}$ in the transition from vegetative to reproductive growth in Chrysanthemum morifolium Ramat. J. Hort. Sci. Biotech. 84: 447-453.

Lifschitz, E., T. Eviatar, A. Rozman, A. Shalit, A. Goldshmidt, Z. Amsellem, J. P. Alvarez and Y. Eshed. 2006. The tomato FT ortholog triggers systemic signals that regulate growth and flowering and substitute for diverse environmental stimuli. Proc. Natl. Acad. Sci. USA 103: 6398-6403.

Mandel, M. A., C. Gustafson-Brown, B. Savidge and M. F. Yanofsky. 1992. Molecular characterization of the Arabidopsis floral homeotic gene APETALA1. Nature 360: 273-277.

Mazier, M., E. Botton, F. Flamain, J. Bouchet, B. Courtial, M. Chupeau, Y. Chupeau, B. Maisonneuve and H. Lucas. 2007. Successful gene tagging in lettuce using the Tnt1 retrotransposon from tobacco. Plant Physiol. 144: 18-31.

Michaels, S. D. and R. M. Amasino. 1999. FLOWERING LOCUS $C$ encodes a novel MADS domain protein that acts as a repressor of flowering. Plant Cell 11: 949-956.

Notaguchi, M., M. Abe, T. Kimura, Y. Daimon, T. Kobayashi, A. Yamaguchi, Y. Tomita, K. Dohi, M. Mori and T. Araki. 2008. Long-distance, graft-transmissible action of Arabidopsis FLOWERING LOCUS T protein to promote flowering. Plant Cell Physiol. 49: 1645-1658.

Oda, A., T. Narumi, T. Li, T. Kando, Y. Higuchi, K. Sumitomo, S. Fukai and T. Hisamatsu. 2012. CsFTL3, a chrysanthemum 
FLOWERING LOCUS T-like gene, is a key regulator of photoperiodic flowering in chrysanthemums. J. Exp. Bot. 63: 1461-1477.

Parcy, F., O. Nilsson, M. A. Busch, I. Lee and D. Weigel. 1998. A genetic framework for floral patterning. Nature 395: 561566.

Plessis, A., C. Hafemeister, O. Wilkins, Z. J. Gonzaga, R. S. Meyer, I. Pires, C. Müller, E. M. Septiningsih, R. Bonneau and M. Purugganan. 2015. Multiple abiotic stimuli are integrated in the regulation of rice gene expression under field conditions. eLIFE 4: 1-27. DOI: 10.7554/eLife.08411. $<$ https://elifesciences.org/content/4/e08411/>.

Rédei, G. P. 1962. Supervital mutants of Arabidopsis. Genetics 47: 443-460.

Rottmann, W. H., R. Meilan, L. A. Sheppard, A. M. Brunner, J. S. Skinner, C. Ma, S. Cheng, L. Jouanin, G. Pilate and S. H. Strauss. 2000. Diverse effects of overexpression of $L E A F Y$ and PTLF, a poplar (Populus) homolog of LEAFY/ FLORICAULA, in transgenic poplar and Arabidopsis. Plant J. 22: 235-245.

Ruiz-García, L., F. Madueno, M. Wilkinson, G. Haughn, J. Salinas and J. M. Martínez-Zapater. 1997. Different roles of flowering-time genes in the activation of floral initiation genes in Arabidopsis. Plant Cell 9: 1921-1934.

Ryder, E. J. 1999. Lettuce, endive, and chicory. CABI Publishing Series, Oxford.

Samach, A., H. Onouchi, S. E. Gold, G. S. Ditta, Z. SchwarzSommer, M. F. Yanofsky and G. Coupland. 2000. Distinct roles of CONSTANS target genes in reproductive development of Arabidopsis. Science 288: 1613-1616.

Srikanth, A. and M. Schmid. 2011. Regulation of flowering time: all roads lead to Rome. Cell. Mol. Life Sci. 68: 2013-2037.

Sun, X., Q. Qin, J. Zhang, C. Zhang, M. Zhou, K. Y. Paek and Y. Cui. 2012. Isolation and characterization of the FVE gene of a Doritaenopsis hybrid involved in the regulation of flowering. Plant Growth Regul. 68: 77-86.

Takada, S. and K. Goto. 2003. TERMINAL FLOWER2, an
Arabidopsis homolog of HETEROCHROMATIN PROTEIN1, counteracts the activation of FLOWERING LOCUS T by CONSTANS in the vascular tissues of leaves to regulate flowering time. Plant Cell 15: 2856-2865.

Thompson, H. C. and J. K. Knott. 1933. The effect of temperature and photoperiod on the growth of lettuce. Proc. Amer. Soc. Hort. Sci. 30: 507-509.

Toyomasu, T., H. Kawaide, W. Mitsuhashi, Y. Inoue and Y. Kamiya. 1998. Phytochrome regulates gibberellin biosynthesis during germination of photoblastic lettuce seeds. Plant Physiol. 118: 1517-1523.

Vergara, R., X. Noriega, F. Parada, D. Dantas and F. J. Pérez. 2016. Relationship between endodormancy, FLOWERING LOCUS T and cell cycle genes in Vitis vinifera. Planta 243: 411-419.

Wada, M., Q. Cao, N. Kotoda, J. Soejima and T. Masuda. 2002. Apple has two orthologues of FLORICAULA/LEAFY involved in flowering. Plant Mol. Biol. 49: 567-577.

Weigel, D., J. Alvarez, D. R. Smyth, M. F. Yanofsky and E. M. Meyerowitz. 1992. LEAFY controls floral meristem identity in Arabidopsis. Cell 69: 843-859.

Wigge, P. A., M. C. Kim, K. E. Jaeger, W. Busch, M. Schmid, J. U. Lohmann and D. Weigel. 2005. Integration of spatial and temporal information during floral induction in Arabidopsis. Science 12: 1056-1059.

Wittwer, S. H. and M. J. Bukovac. 1957. Gibberellin effects on temperature and photoperiodic requirements for flowering of some plants. Science 126: 30-31.

Xia, Z., S. Watanabe, T. Yamada, Y. Tsubokura, H. Nakashima, H. Zhai, T. Anai, S. Sato, T. Yamazaki, S. Lu, H. Wu, S. Tabata and K. Harada. 2012. Positional cloning and characterization reveal the molecular basis for soybean maturity locus $E 1$ that regulates photoperiodic flowering. Proc. Natl. Acad. Sci. USA 109: E2155-E2164.

Yamaguchi, S. 2008. Gibberellin metabolism and its regulation. Annu. Rev. Plant Biol. 59: 225-251. 